\title{
1 Robust data storage in DNA by de Bruijn graph-based 2 decoding
}

3 Li-Fu Song ${ }^{1,2}$, Feng Geng ${ }^{3}$, Zi-Yi Gong ${ }^{1,2}$, Bing-Zhi Li ${ }^{1,2^{*}}$, Ying-Jin Yuan ${ }^{1,2^{*}}$

$4 \quad{ }^{1}$ Frontier Science Center for Synthetic Biology and Key Laboratory of Systems

5 Bioengineering (Ministry of Education), Tianjin University, Tianjin 300072,

6 China

$7 \quad{ }^{2}$ School of Chemical Engineering and Technology, Tianjin University, Tianjin

8 300072, China

$9{ }^{3}$ College of Pharmacy, Binzhou Medical University, Yantai 264003, China

10 *Corresponding authors: $\underline{\text { bzli@tju.edu.cn,yjyuan@tju.edu.cn }}$

\section{Abstract}

13 DNA data storage is a rapidly developing technology with great potential due to

14 its high density, long-term stability, and low maintenance cost. The major

15 technical challenges include various errors such as the indels, rearrangements,

16 and strand breaks that frequently arise during DNA synthesis, amplification,

17 sequencing, and preservation. Here, we report the development of a de Bruijn

18 graph-based, greedy path search algorithm (DBG-GPS) aiming to handle such

19 issues. We demonstrate that accurate data recovery could be achieved from 
20 deep error-prone PCR products. The robustness of this method was further

21 verified with one hundred parallel data retrievals from PCR products containing

22 numerous rearrangements introduced by intended unspecific amplifications.

23 With DBG-GPS, we successfully decoded the original information even after

24 treating the DNA at $70^{\circ} \mathrm{C}$ for 65 days. DBG-GPS shows linear decoding

25 complexity and is faster than the multiple alignment-based methods by two

26 orders of magnitude, suited for large-scale data storage.

\section{Keywords}

28 Synthetic Biology, DNA data retrieval, DNA codec system, de Bruijn graph, 29 Fountain codes

\section{Introduction}

31 DNA is the natural solution for the preservation of genetic information of all life 32 forms on earth. Recently, million years old genomic DNA of mammoths was 33 successfully decoded revealing its high stability as an information carrier under 34 frozen conditions ${ }^{1}$. Owing to its stability, high density, and low maintenance 35 cost revealed by recent studies, DNA has been considered an ideal storage 36 medium to meet the challenge of data explosion in the future ${ }^{2-9}$. Frequently 37 occurring errors in DNA synthesis, amplification, sequencing, and preservation, 38 however, challenge the reliability of data storage in DNA. Efforts to solve this 39 problem led to the implementation of a codec system which requires two layers 40 of error correction (EC) codes, the outer layer codes handling the strand 41 dropouts and the inner layer codes dealing with intramolecular errors, ensuring 
42 accurate information readouts ${ }^{10-13}$. Strand dropouts are erasures and

43 therefore they can be well solved by erasure codes, e.g. Fountain, 44 Reed-Solomon (RS) $\operatorname{codes}^{10-12}$. By contrast, the performance of existing inner 45 layer codec systems, e.g. multiple alignment-based methods, is less 46 satisfactory as they are not efficient in correcting indel errors, and in particular, 47 not capable of dealing with rearrangements and strand breaks. Highly robust 48 inner codec systems remain to be explored.

49 Base-shift arises from indel is difficult to handle with traditional EC codes, 50 which detect indels but cannot position them. A two-dimensional coding 51 strategy with traditional EC codes can transform the indel errors into 52 substitutions. In this strategy, a single indel in one dimension will lead to a 53 series of 'substitutions' in another dimension (Fig. S1). Thus, this strategy only 54 works with low indel rates. Increasing indels will lead to an overwhelming 55 number of "substitutions" exhausting the column EC codes ${ }^{8,10}$. Recently 56 developed hash encoded, decoded by greedy exhaustive search (HEDGES) 57 codes can be used to correct higher frequency of indels ${ }^{14}$. Based on the 58 simulation, HEDGES codes require $40 \%$ redundancy codes for correction of $3 \%$ 59 indel errors, which would significantly increase the cost of data writing. Strand 60 rearrangements caused by unspecific amplifications are frequently 61 encountered and could be a potential threat to data integrity especially when 62 massive multiple retrievals from a large oligo pool are required. Currently, 63 people optimize PCR conditions to avoid rearrangements to occur and use gel 64 purification to physically exclude rearranged strands from the data pool. None 65 of the existing methods can be used to correct rearrangements. DNAs are 66 subjected to hydrolysis and degradation ${ }^{12,13}$ under certain conditions and in 
67 long-term storage, highlighting the importance of data stability studies on

68 influences of harsh conditions, such as high temperature or UV exposure, and

69 methods of DNA protection ${ }^{8,14}$. New coding methods that can tolerate

70 fragmented DNA strands are nevertheless critical for enhancing the

71 robustness of DNA data storage.

72 As the synthesis of long DNA is currently impractical, data storage in DNA 73 relies on a large number of fragments. Consequently, data decoding involves

74 the assembly of information pieces carried by individual DNA fragments, a 75 process similar to genome assembly from shotgun sequencing. De Bruijn 76 graph theory uses $k$-mer components to represent sequences. The analogy of 77 genome assembly to data decoding and the success of the application of De 78 Bruijn graph theory to genome assembly ${ }^{20,21}$ prompted us to investigate its 79 potentials in DNA data storage.

80 In this study, we report a strand reconstruction algorithm, de Bruijn 81 Graph-based Greedy Path Searching (DBG-GPS) that allows robust DNA data 82 storage. This algorithm handles indels, rearrangements, and strand breaks by 83 strand reconstruction from multiple, error-rich strand copies, resulting in 84 accurate and rapid data retrievals. Using this method, an up to $10 \%$ high indel 85 rate, intended rearrangements and forced DNA strand breaks were shown to 86 be efficiently corrected. DBG-GPS is faster than the clustering and multiple 87 alignment (CL-MA) based methods in decoding by two orders of magnitudes. 88 The linear decoding complexity revealed by simulation suggests that 89 DBG-GPS could be adapted to large-scale data storage. 


\section{Results}

\section{The great potentials of de Bruijn graph-based decoding}

92 To inspect the potentials of de Bruijn graph theory in DNA data storage, we first

93 analyzed effects of representative substitutions, indels, and strand breaks in

94 de Bruijn graph as shown in Fig. 1a. The simple de Bruijn graph on the right

95 was constructed from the four sequence copies of "AACGGCGGCTGT" on the

96 left with a $k$-mer size of four (Fig. 1a-i). The sequence of "AACGGCGGCTGT"

97 is the path of " $a \rightarrow b \rightarrow c \rightarrow d \rightarrow e \rightarrow c \rightarrow f \rightarrow g \rightarrow h$ " in the constructed graph. A

98 substitution of "G" to "A" in sequence \#2 will lead to a branch path, as shown in

99 red color, and decreased coverages of the correct nodes (Fig. 1a-ii). With

100 traditional EC codes, indels are much more difficult to handle than

101 substitutions due to the base shift issue. The representative base indels in de

102 Bruijn graph, however, are highly similar to substitutions (Fig. 1a-iii). Strand

103 breaks also cause serious problems for traditional EC codes. With de Bruijn

104 graph, the strand breaks only reduce the coverages of the correct nodes,

105 without changes the graph structure. A greedy path search process, in

106 combination with a path recognition mechanism, could decode the correct

107 strand. In this way, the errors are transformed into a path search and selection

108 problem by de Bruijn graph theory. With this mechanism, as long as the correct

109 path, e.g. " $a \rightarrow b \rightarrow c \rightarrow d \rightarrow e \rightarrow c \rightarrow f \rightarrow g \rightarrow h$ " in Fig. 1a-i, is preserved, the

110 corresponding DNA strand can be decoded theoretically. Thus, the robustness

111 of the correct path determines the potentials of the graph theory-based

112 decoding. 
113 Clearly, the sequence error rates and copy number together determine the

114 robustness of a strand path in de Bruijn graph. To further investigate how the

115 sequence copies and error rates affect path robustness, we simulated DNA

116 sequences with different error rates. Variant numbers of the simulated

117 error-containing sequences were utilized for de Bruijn graph construction and

118 path integrality analysis (Fig. 1b, c). The integrality of the correct path was

119 tested by checking the existence of all $k$-mers in this path in the constructed

120 graph. The rate of the sequences with a complete path in the graph was

121 defined as 'maximal strand decoding rate' $\left(\boldsymbol{S}_{\boldsymbol{m}}\right)$, which stands for the maximal

122 strand decoding rate achievable by an actual de Bruijn graph-based decoder.

123 Sequences with different types of errors were separately simulated. Twenty

124 copies of sequences in a length of $320 \mathrm{bp}$ containing different types and rates

125 of errors were estimated for $\boldsymbol{S}_{\boldsymbol{m}}$ values. As shown in Fig. 1b, the $\boldsymbol{S}_{\boldsymbol{m}}$ values as

126 expected are inversely related to the error rates but are similar regardless of

127 the error types at the same error rates. Importantly, with merely twenty

128 sequence copies, the sequence path in de Bruijn graph shows high robustness

129 with an error rate of $5 \%$, a common error level in reported DNA data storage

130 studies $^{11,12,15}$. In practice, emerged errors are mixtures of different types.

131 Therefore, we decided to investigate the sequence path robustness by

132 simulations with sequences containing mixed errors. The simulations were

133 performed with variable total error rates from $1 \%$ to $10 \%$, among which the

134 errors are consisted of substitutions (50\%), insertions, and deletions (25\%

135 each), and variable strand copies from 5 to 100. As shown in Fig. 1c, even

136 with a high error rate of $10 \%, S_{m}>0.99$ can still be obtained with sequence

137 copies of 60 only (red dashed line), revealing the great potentials of de Bruijn

138 graph-based decoding. 
140 The decoding process requires three basic steps, de Bruijn Graph construction, 141 greedy path search, and path recognition. The de Bruijn graph size is 142 dependent on the total number of correct $k$-mers plus noise $k$-mers. The

143 number of correct $k$-mers is fixed for the storage of any given volume of 144 information. The number of noise $k$-mers becomes a determinant with a larger 145 number increasing de Bruijn graph size and possible paths, leading to longer 146 path search time. Hence, we propose to include an additional step to exclude 147 noise $k$-mers to reduce search time, illustrated in Fig. 2a.

148 Elimination of noise $k$-mers decreases the greedy path search 149 complexity

150 With simulations, about thirty times enlargement of the de Bruijn graph size 151 was observed when $4 \%$ random errors were introduced into 10,000 DNA 152 strands with 100 sequence copies each (Fig. S2a). To quantify the noise $153 k$-mers in a de Bruijn graph, we introduce an indicator $K_{n}$, which is the ratio of 154 noise $k$-mers and correct $k$-mers in a de Bruijn graph. Clearly, more strand 155 copies and errors will introduce more noise $k$-mers to the de Bruijn graph, 156 resulting in a larger $K_{n}$ value. Monte Carlo simulations show a quick increase 157 of $K_{n}$ by increasing either the error rate or the sequence copies (Fig. S2a). 158 The number of branch paths and decoding time increase exponentially with 159 error rates or strand copy numbers (Fig. S2b).

160 DNA data operations introduce a large number of errors. The occurrence of 161 each error is however a low probability event. Therefore, the coverage of noise 
$162 k$-mers is generally much lower than that of the correct ones. Elimination of the

$163 k$-mers with only one occurrence significantly reduced branch paths not

164 affecting the integrity of correct paths (Fig. S2b). We next attempted to

165 mathematically describe the validity of using coverage as an indicator to

166 recognize noise k-mers. Monte Carlo simulations were performed with 10,000

167 DNA strands, in which a Poisson model was employed for the simulation of the

168 copy number distribution of each strand. The expectation value $(\lambda)$ of strand

169 copies was pre-set to be 50 and the error rate being $4 \%$. The resulting

170 coverage distribution of noise $k$-mers and correct $k$-mers were further analyzed.

171 A coverage cut-off of 8 was found effective in the removal of most noise $k$-mers

172 (Fig. S2c).

173 Path recognition by CRC codes and both-way search strategy

174 Noise $k$-mers cannot be completely removed solely by the elimination of low

175 coverage $k$-mers. Branch paths still exist due to the remaining noise $k$-mers. $A$

176 method to recognize the correct path is thus required. Traditional EC codes

177 including cyclic redundancy check (CRC), RS, and hash codes are choices for

178 this purpose. We failed to find a proper hash function that can generate short

179 keywords. RS codes, optimized for correcting erasures, are not suited for path

180 recognition, which does not involve erasures. CRC codes were chosen

181 because of the efficiency of these codes in error detection instead of error

182 correction. The effectiveness of $\mathrm{CRC}$ codes in path recognition was well

183 demonstrated by intensive (one million) Monte Carlo simulations.

184 To further reduce the greedy path search time, we proposed a both-way 185 search strategy that allows simultaneous search from both ends of DNA 
186 strands. In this strategy, an additional 'anchor' element was designed for each

187 DNA strand. The anchor corresponds to the index located on the other end of 188 the DNA strand (Fig. 2b), and is generated from the index by a specific 189 function. A given index processed by the function generates a specific number, 190 represented by the anchor sequence. For proof-of-concept, the pseudorandom 191 function of Python is used for the generation of the anchors from the indexes 192 as seeds. Pseudorandom function generates an identical 'random' value with 193 the same seed. Thus, informed with indexes, the greedy path search engine 194 calculates corresponding anchors, and carries out the both-way search 195 strategy. The both-way search strategy significantly reduced the decoding time, 196 with effects more obviously observed when sequence error rates are high. Up 197 to $60 \%$ reduction was evident with an error rate of $10 \%$ and a copy number of 198 100, while decoding accuracy was well maintained (Fig. S3).

199 Integration of DBG-GPS with outer erasure codes and 200 performance test

201 DBG-GPS is designed as an inner decoding mechanism for the error-free 202 reconstruction of DNA strands. It can be easily combined into the decoding 203 process of outer erasure codes, e.g. fountain codes or RS codes, as shown by 204 Fig. 2c. Python implementation of DBG-GPS using fountain codes as outer 205 codes is available at 206 https://switch-codes.coding.net/public/switch-codes/DNA-De-Bruijn-Decoding/ 207 git/files.

208 To systematically analyze the decoding accuracy of our Python implementation 209 of DBG-GPS, Monte Carlo simulations of 10,000 DNA strands (each strand 
210 with 60 copies) containing various error rates ranging from $1 \%$ to $10 \%$ were

211 performed and the results were subjected to decoding using the Python 212 implementation and even at $10 \%$ of error, decoding accuracy was still 213 remarkably satisfactory with the overall $S_{r}$ higher than $96.7 \%$ (Tab. S2). We 214 next tested the decoding speed of DBG-GPS in comparison with CL-MA based 215 methods. To the best of our knowledge, the CL-MA based methods are the 216 only methods that have been used to calculate the consensus sequence from 217 multiple error-rich strand copies $^{12,17}$. To compare the decoding speed of 218 DBG-GPS with CL-MA based methods, DNA fountain codes were used to 219 generate 10,000 DNA droplets and fifty sequence copies for each droplet. 4\% 220 errors (2\% substitutions, $1 \%$ insertions and $1 \%$ deletions) were randomly 221 introduced. A total of 500,000 error-rich sequences were then subjected to 222 strand decoding by DBG-GPS and CL-MA respectively. Considering the 223 complexity of the clustering process for the CL-MA-based decoding, we fed the 224 Muscle $3.8 .31^{16}$ program with pre-clustered reads. For decoding speed 225 comparison, estimation based on the previously reported clustering time in 226 literature was used ${ }^{14}$. Since the multiple alignment process was performed by 227 a standalone program (Muscle $3.8 .31^{16}$ ), there will be 10,000 times of IO (input 228 and output) with the file system during strand decoding by CL-MA. To estimate 229 the 10 time, one sequence of the DNA droplet was repetitively submitted to 230 Muscle for multiple alignment analysis for 10,000 times. This process is 231 iterated three times and the average time is subtracted from CL-MA based 232 decoding time. The $k$-mer counting step of DBG-GPS was performed with 233 JellyFish 2.3. As shown in Tab. 1, DBG-GPS shows 103 times faster than the 234 CL-MA based methods. Importantly, in the range of data scale from 10 to 235 10,000 DNA droplets, DBG-GPS exhibited linear decoding complexity (Fig. 
236 S5), suggesting that this method is suited for large-scale data storage.

237 Large-scale simulations were then used to assess the capacity of DBG-GPS 238 with big data sets. An Ubuntu server 20.10 installation disk image ( $1 \mathrm{~GB})$ 239 was used as the input file to generate DNA droplets. Each DNA droplet was set 240 to carry 60 bytes of information with a total DNA length of 316bp, around the 241 technical limits of current DNA synthesis technologies. Ten million DNA 242 droplets, representing a data scale of 600 megabytes (MB) were simulated. 243 With an expected copy number of fifty, error rate of $4 \%$ (approximately $2 \%$ 244 substitutions, 1\% insertions, 1\% deletions), a 155GB sequence file was 245 generated. Loading the k-mer counting results of Jellyfish on a computation 246 workstation equipped with 640GB memories, the Python script encountered 247 memory limitations. We had to reduce the DNA droplet number to five million, 248 representing a data size around $300 \mathrm{MB}$. Preliminary decoding simulations on 249 this data scale revealed a problem of long decoding time. Detailed analysis 250 showed that this is due to repeated presentations of same $k$-mers located in 251 different strands. We then solved the problem using a combination of two 252 strategies, with the first employing a filtering process during the production of 253 DNA droplets to exclude seriously entangled DNA strands, and the second 254 setting an upper boundary for path number in greedy path search. The greedy 255 path search engine aborts search if the number of found paths exceeding this 256 boundary. Five million DNA droplets were simulated three times independently. 257 On average, 4,971,880 DNA droplets $\left(S_{r}=99.44 \%\right)$ were recovered, proving 258 the great capacity of DBG-GPS. 


\section{Experimental verification of the robustness of DBG-GPS}

260 To verify the robustness of DBG-GPS, a $314 \mathrm{~KB}$ zipped file, including two text

261 files and four jpeg pictures, was encoded into 12,000 DNA strands, each in a 262 length of $200 \mathrm{bp}$ using fountain as outer codes. Each strand encodes thirty 263 bytes of data, three bytes of CRC codes, four bytes of index codes, and four 264 bytes of anchor codes (Fig. 2b). The overall strand redundancy was designed 265 as $10.5 \%$ based on the expectation of more than $92 \%$ of the strands could be 266 correctly decoded. A final concentration of $2 \mathrm{ng} / \mu \mathrm{l}$ designed DNA strands were 267 synthesized by Twist Bioscience (Fig. 3a), and used as templates for PCR 268 amplification and data retrievals. Different types of errors, including indels, 269 substitutions, rearrangements, and strand breaks were intentionally introduced 270 by three harsh experiments (Fig. 3b-d), followed by decoding to examine the 271 robustness of DBG-GPS.

\section{Data retrievals with deep error-prone PCR products}

273 A serial of six error-prone PCR (ePCR), with each reaction containing 30 274 thermal cycles, was performed using an error-prone PCR Kit to amplify the 275 ssDNA library introducing large numbers of substitution errors, estimated 8 276 errors per 1,000 bp per amplification cycle base on the manufacturer's 277 instruction. All six ePCR products, ePCR\#1-6, were sequenced by Illumina 278 technology. Due to unspecific amplifications, in addition to substitution errors, 279 ePCR\#2-6 products contain DNAs of different sizes, evident by a smear from 280100 to 4,000 bp on agarose gel (Fig. 4a). As some of these DNA sequences 281 were too long to be sequenced by Illumina platform, these PCR products were 282 digested with DNase I to a target length of 200 bp. After digestion, all 
283 fragments were collected for sequencing. The sequencing raw reads were 284 subjected to DBG-GPS based decoding.

285 Regardless of rough amplification conditions expected to introduce a large 286 number of errors, strikingly the $S_{m}$ values of all six samples were higher than $28799 \%$ (Fig. 4b), with the $S_{m}$ values of ePCR\#1-3 being 100\%, indicating perfect 288 reservation of all strand paths even after 90 cycles of ePCR. $S_{r}$ values in a 289 range of $93.2-99.8 \%$ were obtained for the six samples, ensuring reliable data 290 decoding by the outer fountain codes. Decrease of $S_{r}$ was observed with 291 increased ePCR cycles. More PCR errors were expected to increase branch 292 paths, raising the necessary cutoff for noise $k$-mers elimination. More correct $293 k$-mers were consequently discarded by mistake, resulting in a lower $S_{r}$ value.

294 To further test the robustness of DBG-GPS, sequencing data with low 295 coverage ranging from 1 to 256 was obtained by random sub-sampling on the 296 sequencing results of ePCR\#1 and ePCR\#2. The $k$-mer dropout rates $\left(K_{d}\right)$, 297 noise $k$-mer indicator $\left(K_{n}\right)$ and strand recovery rate $\left(S_{r}\right)$ were calculated and 298 plotted against coverage values (Fig. 4c). As expected, with the same 299 coverage, both $K_{n}$ and $K_{d}$ values of ePCR\#2 are always higher than 300 ePCR\#1, consistent with accumulating errors in each additional round of 301 ePCR. Upon increasing coverage, the $K_{n}$ values of both samples reflecting 302 the numbers of noise $k$-mers increased quickly, while the $K_{d}$ values reflecting 303 the dropouts of correct k-mers decreased quickly. As shown in Fig. 4c, the two 304 samples can be reliably decoded at coverage as low as 16 and 24 . 


\section{Multiple data retrieval and accelerated aging test}

306 Multiple data retrievals from storage media are required in practice. Thus, data

307 robustness sustainable during multiple retrievals needs to be tested for our

308 algorithm. Till now, only a limited number $(<10)$ of multiple retrievals from

309 high-quality PCR products have been reported for DNA data storage. To mimic

310 the required multiple retrievals in practice and further demonstrate the

311 robustness of our method, 100 times parallel data retrievals from

312 PCR-amplified ssDNA library were carried out (Fig. 3), in which massive

313 unspecific amplifications indicated by smears from 100 to 8,000 bp on agarose

314 gel were observed (Fig. 5a). The $S_{m}$ values of all retrievals were still

315 remarkably high in the range from $99.44 \%$ to $99.78 \%$ (Fig. 5b). The $S_{r}$ values

316 ranged from $98.45 \%$ to $99.02 \%$, ensuring accuracy in these data retrievals.

317 The CL-MA based codec systems highly rely on intact DNA strands for 318 decoding, whereas DBG-GPS relies on short $k$-mers. Thus DBG-GPS likely

319 tolerates DNA degradation. To test this hypothesis, accelerated aging 320 experiments were performed as shown in Fig. 3c. The master pool was first 321 PCR-amplified followed by incubation at $70^{\circ} \mathrm{C}$ for a prolonged period of time up 322 to 65 days. Agilent 2100 Bioanalyzer was used to analyze the samples. A 14 323 days of incubation caused the complete disappearance of the 200bp peak 324 prominent in the original sample, indicating that the integrity of the DNA strand 325 has been severely damaged (Fig. S6). Such degree of degradation would 326 have made it impossible to recover the encoded data using the previously 327 reported codec systems. By contrast, DBG-GPS based decoding enabled high 328 strand recovery rates, ensuring accurate data retrievals from all samples that 
329 have been heat-treated for $7,14,30$ and even 65 days. A high $S_{r}$ value of $33098.18 \%$ was obtained with the sample treated at $70^{\circ} \mathrm{C}$ for 65 days (Fig. 5c).

331 This $S_{r}$ value is only slightly lower than that of the control stored at $-20^{\circ} \mathrm{C}$ $332(99.40 \%)$. Data retrievals from accelerated aged DNAs have been 333 reported ${ }^{10,18,19}$. In these studies, the authors tested the effects of different 334 methods for the preservation of data encoded DNA, among which embedment 335 in silicon beads was shown to be the best allowing recovery of information 336 after treatment at $70^{\circ} \mathrm{C}$ for 7 days. According to the authors, this was thermally 337 equivalent to storing information on DNA at $9.4^{\circ} \mathrm{C}$ for 2000 years ${ }^{10}$. Based on 338 this estimation, our DBG-GPS algorithm can be used to retrieve data from a 339 DNA solution preserved at $9.4^{\circ} \mathrm{C}$ for 18,571 years.

\section{Discussion}

341 Data robustness, density and low-cost are important parameters, determining 342 the technical advancements of a storage medium. In general, higher data 343 robustness requires more redundancy codes, which decrease information 344 density and cost effectivity. Based on information theory, redundancy codes in 345 the form of "data repetition" are unfavorable since production of additional data 346 copies on traditional media is cost-ineffective and time-consuming. By contrast, 347 data repetition in the form of DNA strand copies can be obtained inexpensively 348 and speedily. The traditional definition of "coding efficiency" needs to be 349 adapted to DNA data channel. With current technologies, producing a single 350 copy of DNA data is not even doable. It has been reported that serial dilutions 351 to reduce the copy numbers of information-bearing DNA strands till the 352 average copy number below ten resulted in massive strand dropouts, making 
353 reliable data retrieval impractical ${ }^{20}$. Even if a single copy of DNA data is

354 obtainable, it may be damaged due to DNA degradation under certain 355 conditions or during long-term storage. Taken together, the design for DNA 356 storage should be based on the reality and necessity, the presence of more 357 than one copies of DNA strands, and not too many copies (below 100) to 358 maintain high information density. In other words, "multicopy" is crucial for the 359 robustness of DNA data storage and should ideally be maintained in a 360 reasonable range. A new theory to design EC codes taking this unique 361 "multicopy" feature into consideration will help solving the main technical issue 362 in DNA storage, the error-free decoding of DNA sequences in a completely 363 different fashion.

364 To the best of our knowledge, CL-MA based methods are the only reported 365 methods used for DNA decoding from error-containing multicopies. These 366 methods are computation-intensive, and are not robust when dealing with 367 strand rearrangements and breaks. In this study, we developed a strand 368 reconstruction algorithm based on the de Bruijn graph theory and tested its 369 robustness. We were able to accurately and rapidly decode DNA data from 370 error-rich strand copies using this algorithm. The errors include indels, 371 rearrangements and strand breaks. It is worth noting that the encoded 372 information from a DNA solution that has been incubated at $70^{\circ} \mathrm{C}$ for 65 days 373 can be accurately recovered with our algorithm. The degree of damage to DNA 374 under this heating condition was estimated to be equivalent to that under $9.4^{\circ} \mathrm{C}$ 375 for more than 18,000 years $^{10}$. Such unprecedented robustness highlights the 376 importance of our method and suggests the great potential of de Bruijn graph 377 theory in DNA data channel. Our algorithm is presumably compatible with a 
378 variety of DNA preservation, e.g. silicon beads $^{10}$, nanoparticles ${ }^{18}$, and alkaline

379 salts $^{19}$, to further enhance data stability.

380 The Python codes implemented in this study use a simple hash dictionary for

381 the retrieval of $k$-mers. $\mathrm{C}++$ implementation with a more advanced $k$-mer data 382 structure ${ }^{21,22}$ is expected to significantly reduce the memory cost, as well as 383 decoding time. In data decoding from 50 sequence copies containing $4 \%$ 384 errors, the performance of DBG-GPS is superior to that of the CL-MA based 385 methods in speed by one hundred fold. A higher ratio of noise $k$-mers is 386 expected to decrease decoding speed and accuracy with the DBG-GPS 387 method. Future incorporation of EC codes to help identify and exclude noise $388 k$-mers prior to the greedy path search is expected to further improve the 389 decoding speed and accuracy, which is particularly important in the extreme 390 case of high error rate.

391 Methods

392 Library design, PCR and Sequencing

393 A DNA library of 12,000 oligonucleotides in length of $200 \mathrm{nt}$ was synthesized 394 by Twist Biosciences. Error-prone PCR was performed with Controlled 395 Error-prone PCR Kit, TIANDZ, CAT\#:160903-100. Thermo cycle parameters 396 were $94^{\circ} \mathrm{C}$ for 3 mins, $94^{\circ} \mathrm{C}$ for $1 \mathrm{~min}, 45^{\circ} \mathrm{C}$ for $1 \mathrm{~min}, 72^{\circ} \mathrm{C}$ for $1 \mathrm{~min}, 30$ cycles. 397 Six serial error-prone PCR were performed to introduce high rates of errors. 398 The first round ePCR utilized $0.6 \mu \mathrm{l}$ samples of the master pool as templates. 399 The five following ePCR use $1 \mu$ products of the previous round ePCR as 400 templates. All PCR experiments were performed with the following primers: P1 
401 5'-CCTGCAGAGTAGCATGTC-3', P2 5'-CGGATGCATCAGTGTCAG-3'. The

402 PCR products were sequenced by Tianjin Novogene Sequencing Center \&

403 Clinical Lab. Sequencing libraries were generated with purified PCR products

404 using Illumina TruSeq DNA PCR-Free Library Preparation Kit (Illumina, USA)

405 following manufacturer's recommendations and index codes were added. For

406 the products of the second-round error-prone PCR with very large DNA

407 strands, DNase digestion is applied to break the long strands into small

408 fragments around 200 300 bp. After digestion, all fragments are collected for

409 further library construction using the protocol as just described. The library

410 quality was assessed on the Qubit@ 2.0 Fluorometer (Thermo Scientific) and

411 Agilent Bioanalyzer 2100 system. All the libraries were sequenced on Illumina

412 HiSeq platform and 150 bp paired-end reads were generated. For preparation

413 of the 100 PCR samples for parallel retrievals, we first performed one round of

414 PCR amplification with Taq DNA polymerase with a total volume of $100 \mu \mathrm{l}$.

415 Then, 100 parallel PCR amplification was performed using $1 \mu$ of the first

416 round PCR reaction mixtures without purification. The purification step is

417 removed in purpose of enhancing unspecific amplification. The 100 PCR

418 samples were then sequenced by Illumina sequencing platform. All the Taq

419 DNA Polymerase based PCR amplifications, except the error-prone PCRs,

420 used Vazyme 2X Rapid Taq Master Mix (CAT\#: P222-03).

\section{$421 \boldsymbol{k}$-mers counting and elimination of low coverage $\boldsymbol{k}$-mers}

422 The python implementation provided in this study used a simple hash 423 dictionary structure for counting of k-mers, which is not memory efficient. Thus, 424 although the scripts implemented with functions to read the common 
425 nucleotide sequence files, i.e. FastQ or FastA, and count the $k$-mers

426 accordingly, for large data sets, we strongly suggest to use a professional

$427 k$-mer counting system, e.g. Jellyfish ${ }^{21}$, which is much more memory efficient

428 and supports multi-cores. To count $k$-mers by the python scripts, please call

429 the following functions of DeBruijnGraph() object: for reading and counting of

430 FastQ file, use DeBruijnGraph-openFastQ('file name'); for reading and

431 counting of Fasta file, use DeBruijnGraph-openFasta('file name').

432 For elimination of low coverage $k$-mers, call function DeBruijnGraph-

433 remove_low_cov_kmers $(n)$, then all $k$-mers with coverages lower than $n$ will be

434 removed. For usage of Jellyfish for counting of $k$-mers and dumping of $k$-mers

435 satisfying the criteria, please refer to the Jellyfish manual, which is available at

436 (http://www.cbcb.umd.edu/software/jellyfish/). The dumped k-mers can be

437 introduced to the DeBruijnGraph() object using DeBruijnGraph-openDump('file

438 name') function for further decoding test.

439 Setting of $k$-mer size

440 Obviously, with a specific $k$-mer size, the data scale can be decoded is limited.

441 For example, it is clear that only small amount of data can be decoded with an

442 extremely short $k$-mer size of three. However, the mathematical answer to this

443 question is yet unsolved. In de Bruijn Graph theory, for each k-mer, the front

$444 k-1$ bases were used for positioning (i.e. indexing), and the one base at the

445 terminal was used for extension of path, i.e. for encoding of data. Thus, we

446 estimate the decoding capacity $(p)$ of $k$-mers with a specific size of $k$ as

447 follows: 


$$
p=4^{k-1} \times 2 \text { bits }
$$

448 Where $4^{k-1}$ is the combination number of possible indexes with a length of $449 k-1,2$ bits stands for the encoding capacity of one base at the tail. Based on 450 this formula, the decoding capacities of different $k$-mer sizes were calculated 451 and listed in Tab. S3. As shown in the table, the decoding capacity is estimated 452 to be around 57.6 PB with a $k$-mer size of 30 , and $6 \times 10^{5} \mathrm{~EB}$ for a $k$-mer size of 45340 , indicating a capable method for large-scale data decoding. Additionally, it 454 should be noticed that, due to the repeated presentations of identical $k$-mers in 455 different DNA strands, the actual data scale can be higher than this estimation 456 theoretically.

457 Details of greedy path search

458 The greedy search process was illustrated in Fig. S4. The details are 459 described as follows: Step 1, Select an index, e.g. 562451, and calculate the 460 anchor value by the Python pseudorandom function using the index value as 461 seed in case of both-way search mode. An anchor value of 47736 is obtained 462 in the case of 562451. Step 2, Encode the index into DNA string based on the 463 corresponding binary string, i.e. $562451=10001001010100010011=$ 464 ATATATGGGATCAGAA. Similarly, the anchor of 47736 is encoded into DNA 465 string of ATATATATGCGTTTGG in case of both way search. The greedy path 466 search starts with ATATATGGGATCAGAA as an initial path. Similarly, the tail 467 search start with ATATATATGCGTTTGG as an initial path in the case of 468 both-way search. Step 3. Judgement step. This step is for the selection of an 469 end with few path numbers for extension of paths. Step 4, Extension of paths 470 on the end with fewer path numbers. Step 5, Judgment step. This step is to 
471 judge if the path search has finished. Step 6, This step is specifically required

472 for both-way search mode. The head paths are fused with tail paths if the

473 overlap sequences are identical. For one-way search, this step is not required.

474 Step 7, Encode the complete paths into binary strings. Perform parity checks

475 and drop the wrong routes using the embedded CRC codes. For other EC

476 codes applied, use the checking mechanism correspondingly. Step 8,

477 Judgement step. Check if there is only one path passed the CRC checking. If it

478 is true, choose it as decoded strands, otherwise, mark a missing strand for this

479 index. If multiple routes or no routes pass the $\mathrm{CRC}$ codes, the 480 searching-based decoding fails. Only in the case of one route passed the CRC 481 checking, the decoding succeeds. Step 9, Judgement step. Judge if the 482 search is finished. If not, iterate step 1 to step 9 until all indexes are searched 483 for possible strands. If yes, finish the inner decoding process. This algorithm is 484 implemented in the deBruijnGraph.py object by Python. The deBruijnGraph() 485 object was implemented to search in both-way mode by default. Switch the 486 value of DeBruijnGraph() ->dual_search to 'False' to active the one-way 487 search mode.

\section{Error analysis in the concept of de Bruijn graph theory}

489 Although distribution profiles of error rate and copy number among different 490 strands shape the DNA data quality, they cannot reflect the data quality 491 straightforwardly. Additionally, the sequence error rate is complicated for 492 accurate accounting. For better estimation of the qualities of DNA data and 493 simplification of the error analysis process in the concept of de Bruijn graph 494 theory, here we introduce two indicators, i.e. $K_{d}$ (in range of 0 to 1 ) and $K_{n}$ 
495 (in range of one to $4^{k}$ ), which together can well define the data quality. The $K_{d}$

496 stands for dropout rate of all correct $k$-mers and $K_{n}$ is the ratio of noise $k$-mers

497 and correct $k$-mers. DNA data with a $K_{d}$ close to 1 and a $K_{n}$ close to 0 stands

498 for high-quality data with a high data coverage rate and low noises. Other than

499 error rate which is complicated for accounting, $K_{d}$ and $K_{n}$ can be easily

500 calculated by the following formulas:

$$
K_{d}=K_{\text {lost }} / K_{\text {ori }}
$$

$$
K_{n}=K_{\text {all }} / K_{\text {corr }}
$$

503 In formula $1, K_{\text {ori }}$ stands for the number of $k$-mers in the original encoded

504 sequences and $K_{\text {lost }}$ stands for the number of $k$-mers that presented in the 505 original encoded sequences but not presented in the sequencing results. In 506 formula 2, $K_{\text {all }}$ stands for the number of $k$-mers in the sequencing results and $507 K_{c o r r}$ stands for the number of correct $k$-mers, i.e. $k$-mers that presented in the 508 original encoded sequences. DNA data with a $K_{d}$ close to 1 and a $K_{n}$ close 509 to 0 stands for a high-quality data with high data integrity and low noises. The 510 maximal strand decoding rate $S_{m}$ is determined by $K_{d}$. With specific $K_{d}$, the 511 actual strand decoding rate $S_{r}$ is affected by the value of $K_{n}$. The greedy path 512 search becomes extremely slow when decoding DNA data with higher $K_{n}$ 513 values, due to too many possible paths for each strand. The DBG-GPS will 514 stop decoding if the path number exceeds a specific threshold. In this 515 proof-of-concept study, we use 100 for fast decoding, 1,000 for high-quality 516 decoding. 


\section{The fountain codes}

518 The outer fountain codes used in this study is modified from

519 https://github.com/dbieber/fountaincode. DNAFountain and DNADroplet

520 objects were implemented for the handling of DNA strings. The assignment

521 mechanism of the random degrees and chunk indexes of the Droplet object

522 was modified to support DBG-GPS based decoding. Luby transform is applied

523 for the generation of random degrees. Specific index encoded in the DNA

524 Droplet is used for the selection of a degree from a pre-generated degree table.

525 This index is also used as seed for the generation of the random strand

526 combinations. The decoding process of the original Glass object has been

527 modified to improve its performance.

\section{Details of the simulation experiments}

529 Simulations for investigation of the potentials of de Bruijn graph were

530 performed with 10,000 DNA strands generated by the fountain codes with a 531 strand length of 320 bp. For de Bruijn graph construction, a k-mer size of 21

532 was applied. The simulation studies on the both-way search strategy are 533 performed with 10,000 DNA droplets. 100 sequence copies with random base

534 errors in rates of $10 \%$. For the large-scale simulations, an Ubuntu Server 20.10 535 live install disk image (Link provided in Supplementary materials) was used 536 as input file for the generation of random DNA droplets. Each DNA droplet was 537 set to encode 60 bytes of data and two bytes of CRC codes. The length of 538 index and anchor are both set to four bytes. Primers of P1 539 5'-CCTGCAGAGTAGCATGTC-3' and P2 5'-CGGATGCATCAGTGTCAG-3' 540 were added to the ends. This will result with a DNA droplet in length of $300 \mathrm{bp}$, 
541 around the theoretical limits of current DNA synthesis technologies. To avoid

542 entangled DNA strands, a filter process that always discards the DNA droplets

543 contains a $k-1$ mer that already presents more than four times was applied to

544 the droplet generating stage. For counting of $k$-mers by Jellyfish 2.3 , a $k$-mer

545 size of 24 , and $k$-mer coverage cut-off of 5 .

\section{Data Availability}

547 All data files and raw sequencing data that support the findings of this study

548 have been deposited in public cloud server. The links are provided in

549 supplementary materials.

\section{Code availability}

551 All the original algorithms proposed in this study were implemented with

552 Python and the source codes are available at

553 https://switch-codes.coding.net/public/switch-codes/DNA-De-Bruijn-Decoding/

554 git/files.

\section{Acknowledgements}

556 This work was supported by National Natural Science Foundation of China

557 under the grants 21621004 to Y.Y. , Seed Foundation of Tianjin University to L.

558 S. and Natural Science Foundation of Shandong Province under the grants

559 ZR2017LC006 to F.G.. The authors would like to thank Dr. Yan Zhang for his

560 great efforts and constructive comments which have helped to improve this

561 manuscript significantly. The authors also would like to thank Dr. Sheng Ye, Dr. 
562 Hao Qi and Dr. Weigang Chen for very helpful suggestions.

563 Competing interests

564 Li-Fu Song, Ying-Jin Yuan and Feng Geng have filed a patent covering the 565 encoding structure and the de Bruijn graph based decoding process (CN 566110190858 A).

\section{Author contributions}

568 LFS and Y.Y. conceived the study. LFS proposed the idea, wrote the Python

569 codes, designed the experiments, performed the simulations, and analyzed

570 the data. FG helped with experiment design and data analysis. ZYG performed

571 the error-prone PCR experiments. BZL and YJY edited the manuscript and

572 supervised the whole work.

\section{References}

574 1. van der Valk, T. et al. Million-year-old DNA sheds light on the genomic

575 history of mammoths. Nature 591, 265-269; 10.1038/s41586-021-03224-9

$576 \quad(2021)$.

577 2. Anavy, L., Vaknin, I., Atar, O., Amit, R. \& Yakhini, Z. Data storage in DNA

578 with fewer synthesis cycles using composite DNA letters. Nature

579 biotechnology; 10.1038/s41587-019-0240-x (2019).

580 3. Koch, J. et al. A DNA-of-things storage architecture to create materials with 
581 embedded memory. Nature biotechnology 38, 39-43;

$582 \quad$ 10.1038/s41587-019-0356-z (2020).

583 4. Zhirnov, V., Zadegan, R. M., Sandhu, G. S., Church, G. M. \& Hughes, W. L.

$584 \quad$ Nucleic acid memory. Nature materials 15, 366-370; 10.1038/nmat4594 585 (2016).

586 5. Church, G. M., Gao, Y. \& Kosuri, S. Next-generation digital information

587 storage in DNA. Science (New York, N.Y.) 337, 1628;

$588 \quad 10.1126 /$ science.1226355 (2012).

589 6. Ceze, L., Nivala, J. \& Strauss, K. Molecular digital data storage using DNA.

$590 \quad$ Nature reviews. Genetics 20, 456-466; 10.1038/s41576-019-0125-3

591 (2019).

592 7. Ping, Z. et al. Carbon-based archiving: current progress and future

593 prospects of DNA-based data storage. GigaScience 8;

$594 \quad$ 10.1093/gigascience/giz075 (2019).

595 8. Chen, W. et al. An artificial chromosome for data storage. National Science 596 Review; 10.1093/nsr/nwab028 (2021).

597 9. Tabatabaei, S. K. et al. DNA punch cards for storing data on native DNA 598 sequences via enzymatic nicking. Nature communications 11, 1742;

$599 \quad 10.1038 /$ s41467-020-15588-z (2020).

600 10.Grass, R. N., Heckel, R., Puddu, M., Paunescu, D. \& Stark, W. J. Robust

601 chemical preservation of digital information on DNA in silica with 
602 error-correcting codes. Angewandte Chemie (International ed. in English)

$603 \quad 54,2552-2555 ; 10.1002 / a n i e .201411378$ (2015).

604 11.Erlich, Y. \& Zielinski, D. DNA Fountain enables a robust and efficient

605 storage architecture. Science (New York, N.Y.) 355, 950-954;

$606 \quad 10.1126 /$ science.aaj2038 (2017).

607 12.Organick, L. et al. Random access in large-scale DNA data storage. Nature 608 biotechnology 36, 242-248; 10.1038/nbt.4079 (2018).

609 13.Gao, Y. et al. Low-Bias Amplification for Robust DNA Data Readout (2020).

610 14.Press, W. H., Hawkins, J. A., Jones, S. K., Schaub, J. M. \& Finkelstein, I. J. 611 HEDGES error-correcting code for DNA storage corrects indels and allows 612 sequence constraints. Proceedings of the National Academy of Sciences of 613 the United States of America 117, 18489-18496;

$614 \quad$ 10.1073/pnas.2004821117 (2020).

615 15.Heckel, R., Mikutis, G. \& Grass, R. N. A Characterization of the DNA Data 616 Storage Channel. Scientific reports 9, 9663; 10.1038/s41598-019-45832-6 617 (2019).

618 16.Edgar, R. C. MUSCLE: multiple sequence alignment with high accuracy 619 and high throughput. Nucleic acids research 32, 1792-1797;

$620 \quad 10.1093 /$ nar/gkh340 (2004).

621 17.Antkowiak, P. L. et al. Low cost DNA data storage using photolithographic 622 synthesis and advanced information reconstruction and error correction. 
$623 \quad$ Nature communications 11, 5345; 10.1038/s41467-020-19148-3 (2020).

624 18.Chen, W. D. et al. Combining Data Longevity with High Storage

625 Capacity_Layer $\square$ by $\square$ Layer DNA Encapsulated in Magnetic Nanoparticles.

626 Adv. Funct. Mater. 29, 1901672; 10.1002/adfm.201901672 (2019).

627 19.Kohll, A. X. et al. Stabilizing synthetic DNA for long-term data storage with 628 earth alkaline salts. Chemical communications (Cambridge, England) 56, 629 3613-3616; 10.1039/d0cc00222d (2020).

630 20.Chen, Y.-J. et al. Quantifying molecular bias in DNA data storage. Nature 631 communications 11, 3264; 10.1038/s41467-020-16958-3 (2020).

632 21.Marçais, G. \& Kingsford, C. A fast, lock-free approach for efficient parallel 633 counting of occurrences of k-mers. Bioinformatics (Oxford, England) 27, 634 764-770; 10.1093/bioinformatics/btr011 (2011).

635 22.Pandey, P., Bender, M. A., Johnson, R., Patro, R. \& Berger, B. Squeakr: an 636 exact and approximate k-mer counting system. Bioinformatics (Oxford, 637 England) 34, 568-575; 10.1093/bioinformatics/btx636 (2018).

638 23.Cyrus Rashtchian et al. Clustering Billions of Reads for DNA Data Storage. 
639 Fig. 1 | The potentials of de Bruijn graph-based decoding. a, 640 Representations of base substitutions and indels in de Bruijn graph. $\boldsymbol{i}$, Simple 641 de Bruijn graph constructed from four copies of "AACGGCGGCTGT" with a $642 k$-mer size of four. The circles stand for the $k$-mers and the numbers inside the 643 circles are coverages of corresponding $k$-mers in all graphs. ii, De Bruijn graph 644 as consequence of one substitution occurring in sequence \#2; iii, De Bruijn 645 graph as consequence of one base insertion on sequence \#2 and one base 646 deletion on sequence \#3; iv, De Bruijn graph involves four strand breaks. b, 647 Estimated maximal strand decoding rates $\left(\boldsymbol{S}_{\boldsymbol{m}}\right)$ of different types and rates of 648 errors with 20 sequence copies; For clarity, the standard derivations are 649 detailed in Tab. S1. c, $\boldsymbol{S}_{\boldsymbol{m}}$ values estimated with different sequence copies and 650 rates of mixture errors (50\% substitutions, $25 \%$ insertions and $25 \%$ deletions).

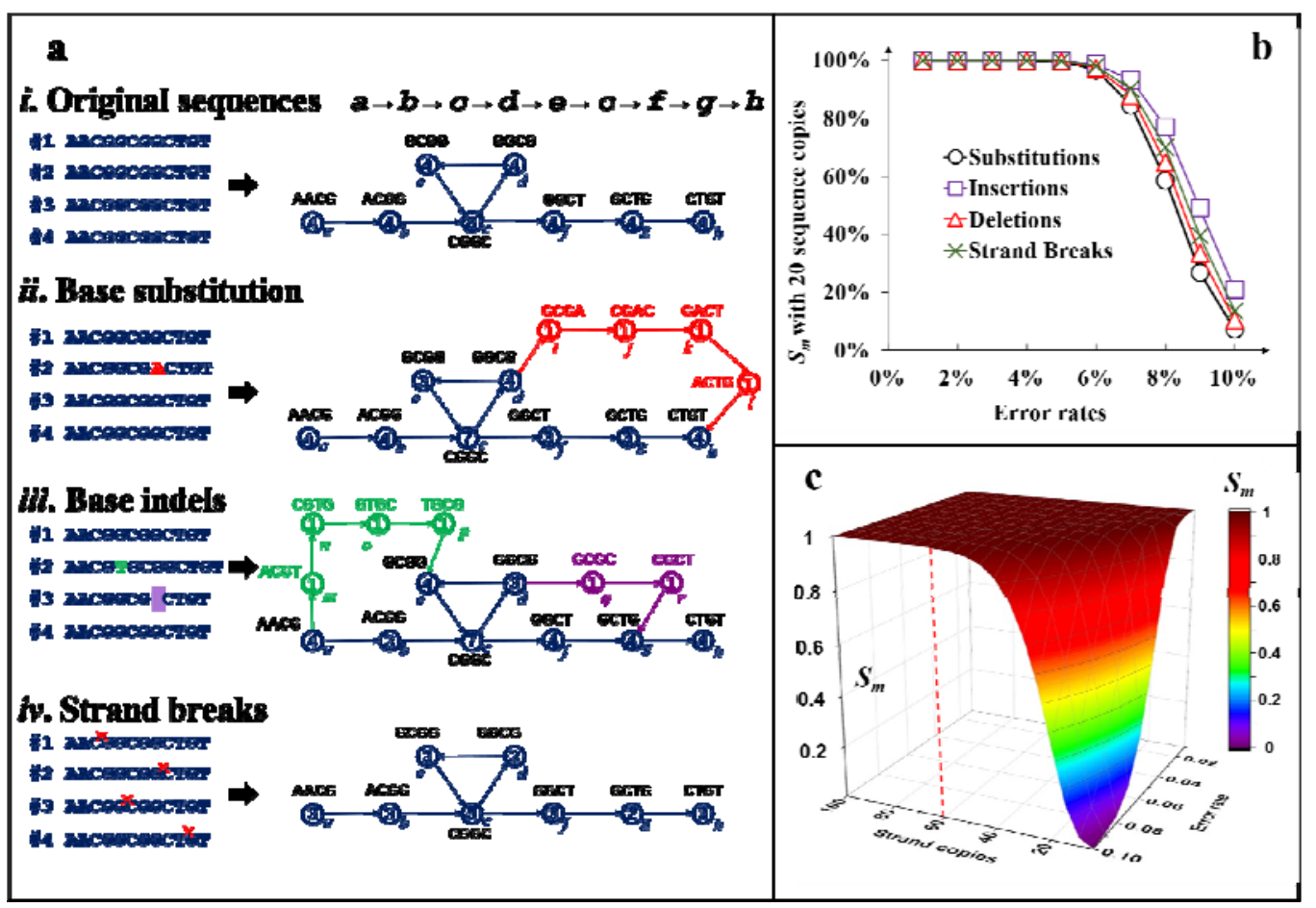


654 Fig. 2 | Proposed four-step framework of DBG-GPS and its integration with outer erasure codes. a, Proposed four-step 655 framework of DBG-GPS. b, Proposed structure of DNA strands for DBG-GPS. There are four major parts including: A1 - The 656 index/seed of the DNA strand; A2 - Data payload for encoding of data; A3 - Cyclic Redundancy Check codes (CRC) for selection of 657 the correct path; A4 - Anchor cods which can be calculated by specific function based on the value of A1. A4, Anchor codes 658 (optional) for both-way search; c, Integration of DBG-GPS with outer erasure codes. DBG-GPS is a full resolution for inner code 659 decoding. 


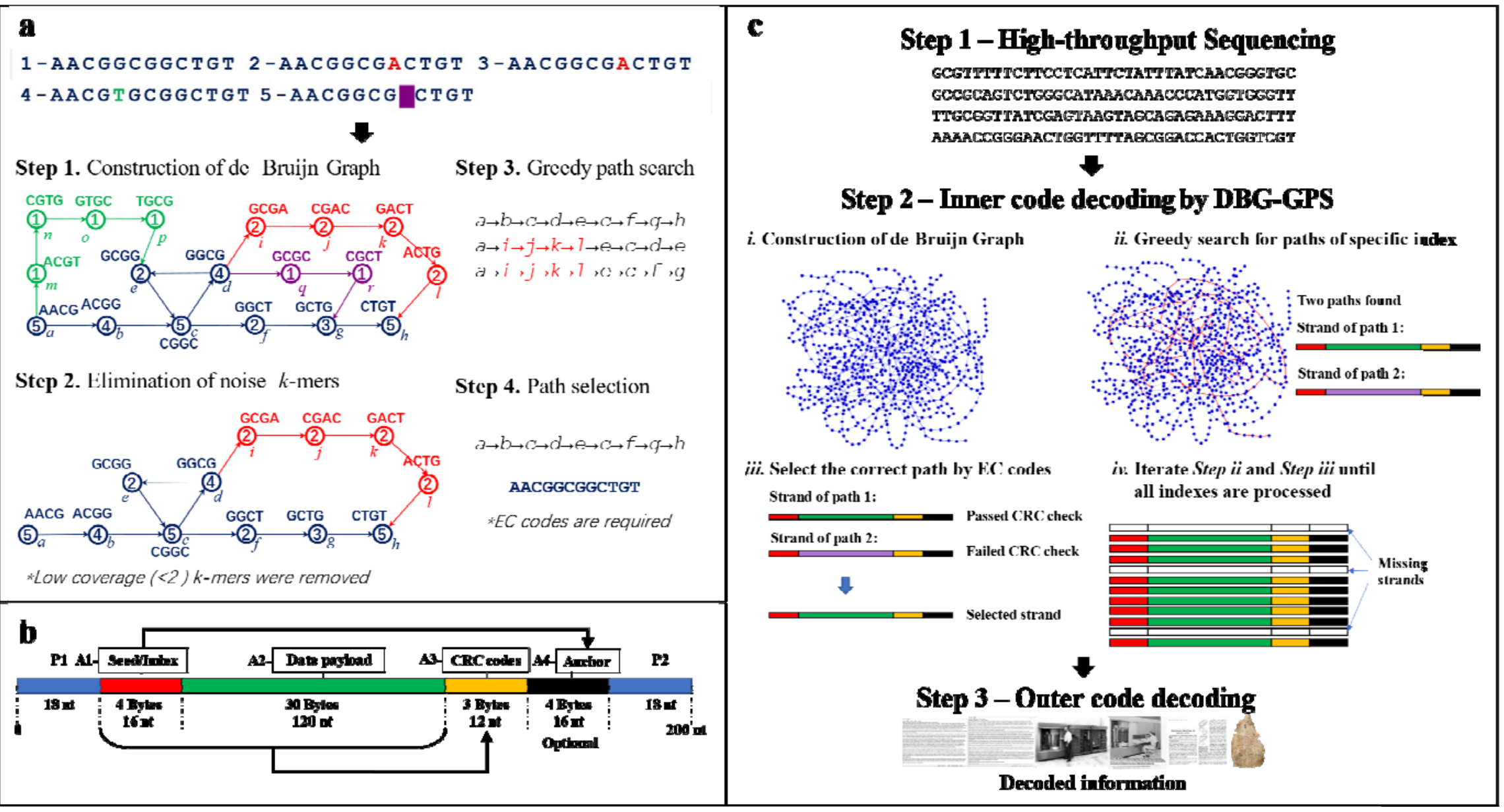


662 Fig. 3 | Experimental verification of the robustness of DBG-GPS. a, A $663314 \mathrm{~kb}$ file is wrote on DNAs to generate a "Master Pool". b, Serial deep 664 error-prone PCR to introduce base errors. c, Multiple data retrievals with 665 intended unspecific amplifications to introduce strand rearrangements. d, 666 Accelerated aging experiments to introduce strand breaks.

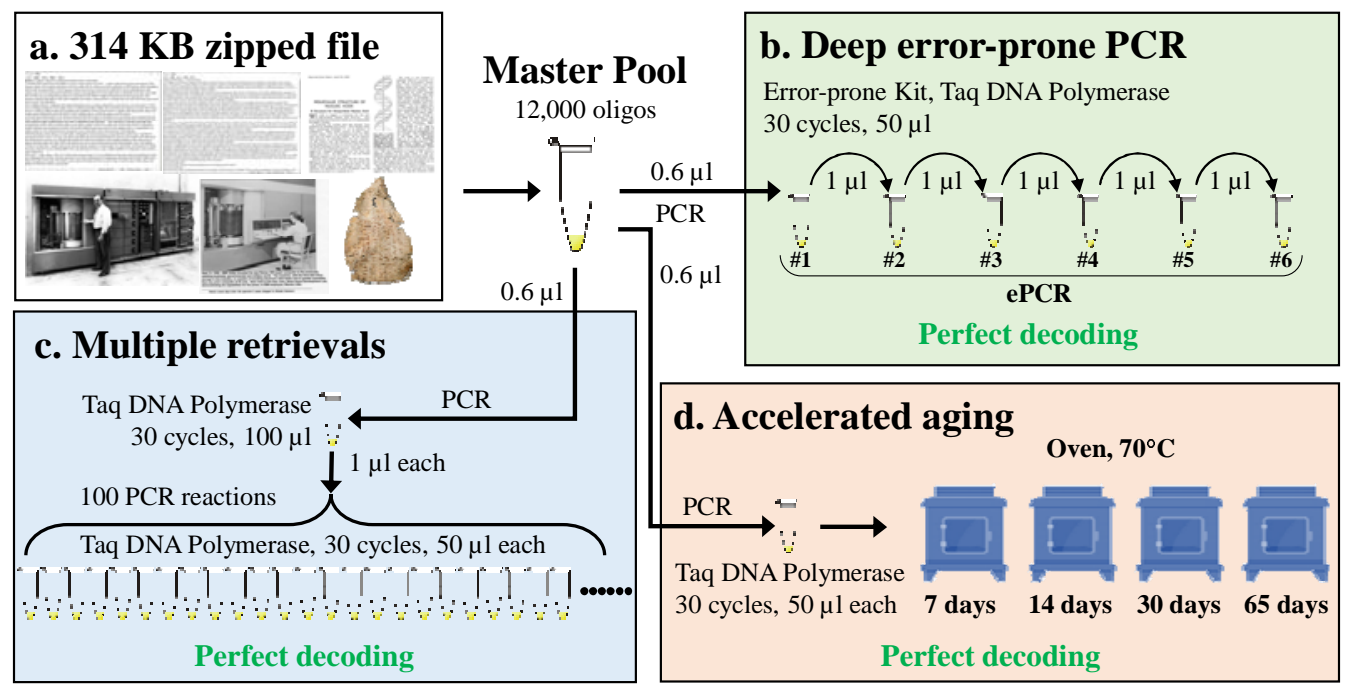


669 Fig. 4 | Reliable data recovery with deep error-prone PCR products. a,

670 Agarose electrophoresis analysis of the serial error-prone PCR (ePCR)

671 products. b, Reliable strand reconstruction with low quality ePCR products.

672 The dashed red line stands for the decoding lower boundary of the outer

673 fountain codes in this case study. c, Low coverage decoding by random

674 sub-sampling. Sequencing reads of ePCR\#1 and ePCR\#2 are used for

675 random sub-sampling individually. For each coverage tested, 12 independent

676 sub-sampling simulations were performed and the averages values were

677 presented. The standard deviations are presented in Tab. S5 for clarity.

678 Coverages in range of 1 to 256 were considered. The average strand recovery

679 rates $\left(S_{r}\right)$, $k$-mer dropout rates $\left(K_{d}\right)$ and $K_{n}$ values were presented. The dashed

680 curves are the strand recovery results without elimination of low coverage

$681 k$-mers. The horizontal dashed red line stands for the decoding limits by the

682 outer Fountain codes.

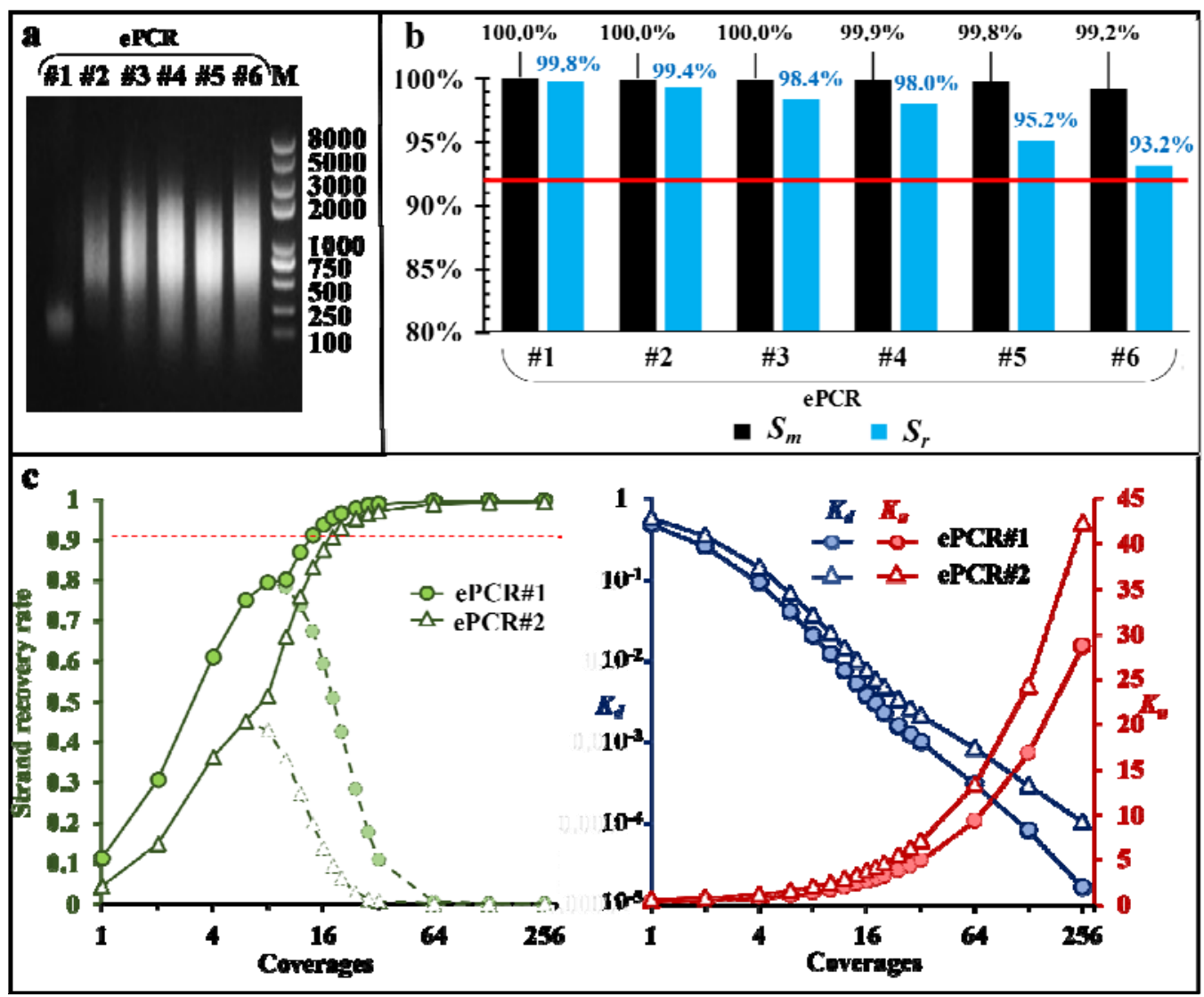


bioRxiv preprint doi: https://doi.org/10.1101/2020.12.20.423642; this version posted April 18, 2021. The copyright holder for this preprint (which was not certified by peer review) is the author/funder. All rights reserved. No reuse allowed without permission.

\section{4}


685 Fig. 5 | Multiple retrieval and accelerated aging tests. a, Agarose 686 electrophoresis analysis of the parallel PCR products for multiple data 687 retrievals. Twenty representatives out of 100 samples are shown. M stands for 688 the DNA Marker. b, Strand decoding results of the 100 multiple retrievals with 689 DBG-GPS. c, Strand decoding rates by DBG-GPS from accelerated aged DNA 690 samples. 


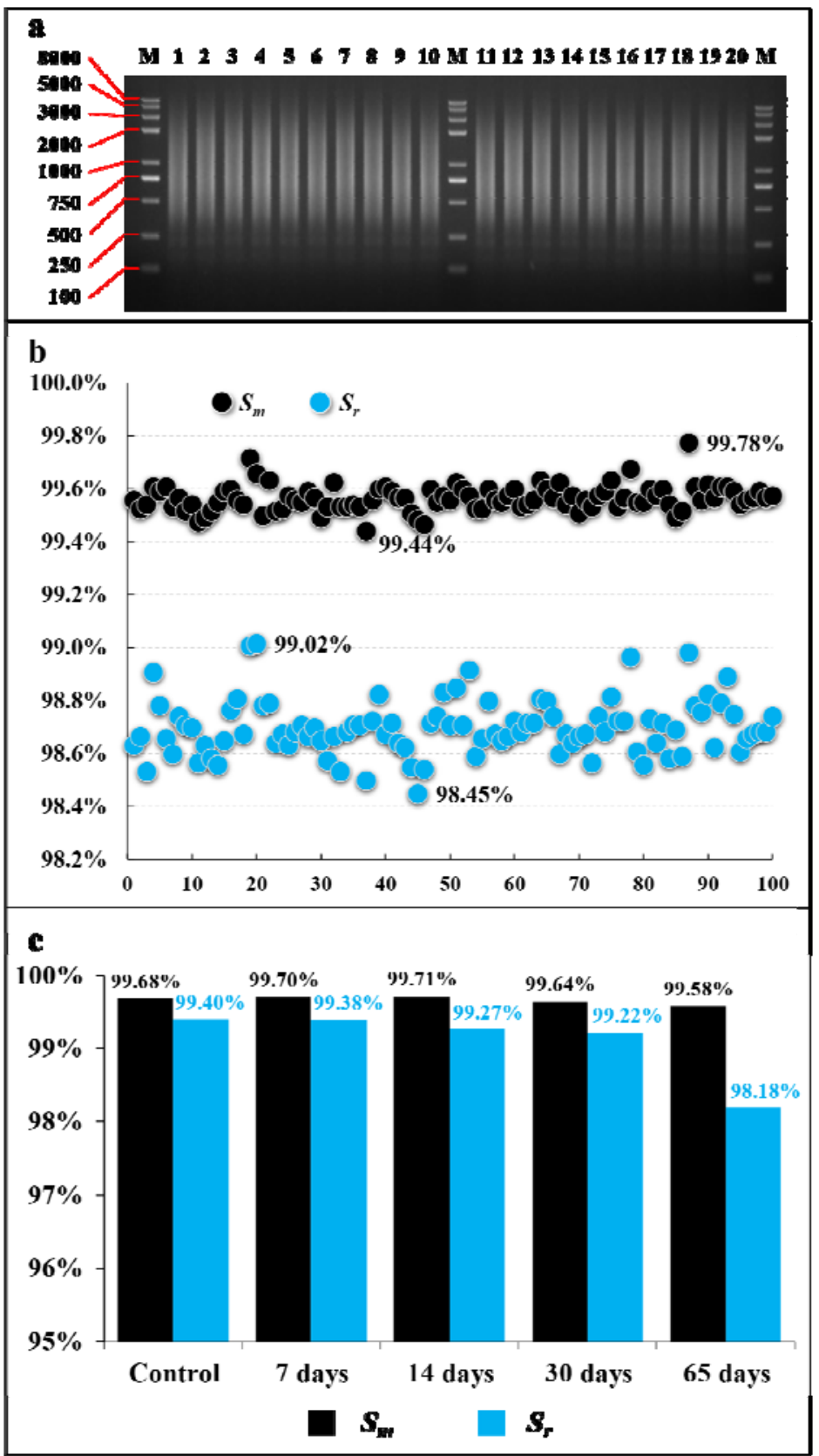


693 Tab. 1 | Comparison of the DBG-GPS and CL-MA based methods in 694 decoding speed.

\begin{tabular}{|c|c|c|c|}
\hline DBG-GPS & & CL-MA & \\
\hline Stages & Time (s) & Stages & Time (s) \\
\hline $\begin{array}{l}\text { k-mer counting } \\
\text { (Jellyfish } 32 \text { threats) }\end{array}$ & $0.63 \pm 0.04$ & $\begin{array}{l}\text { Clustering }^{23} \\
\text { (32 threats) }\end{array}$ & 0.36 \\
\hline \multirow[t]{2}{*}{$\begin{array}{l}\text { Strand decoding } \\
\text { (Python Script) }\end{array}$} & $36.84 \pm 0.91$ & $\begin{array}{l}\text { Multi-alignment } \\
\text { (Muscle }^{16}, \text { IO time } \\
\text { subtracted) }\end{array}$ & $3874.48 \pm 30.82$ \\
\hline & & $\begin{array}{l}\text { Consensus calling } \\
\left(\text { Majority voting }^{13}\right)\end{array}$ & $15.42 \pm 0.40$ \\
\hline Total & $37.47 \pm 0.95$ & Total & $3890.26 \pm 31.22$ \\
\hline
\end{tabular}




\section{Supplementary materials}

696 Fig. S1 | A two-dimensional coding strategy of traditional EC codes

697 handles the base shift problem of indel inefficiently.

698 Fig. S2 | Path search complexity caused by base errors can be

699 remarkably released by elimination of low coverage $k$-mers.

700 Fig. S3 | A both-way search strategy reduces decoding time.

701 Fig. S4 | Details of DBG-GPS algorithm.

702 Fig. S5 | Linear decoding complexity of DBG-GPS.

703 Fig. S6 | Agilent 2100 analysis of the accelerated aged samples.

704 Tab. S1 | Computational estimation of the maximal strand decoding rates

$705\left(S_{m}\right)$ of different types and rates of errors with 20 strand copies.

706 Tab. S2 | Strand decoding performance of four-step DBG-GPS algorithms

707 estimated by Monte Carlo simulations.

708 Tab. S3 | Theoretical decoding capacities of DBG-GPS with different

$709 \quad k$-mers sizes.

710 Tab. S4 | Details of strand recovery results of deep ePCR experiments.

711 Tab. S5 | Details of the sub-sampling simulation results with ePCR\#1 and 712 ePCR\#2. 